\title{
LUT
}

University

\section{Dynamic analysis and control of a Fusion Reactor Vacuum Vessel Assembly Robot}

Moradkhani Shayan, Shabbouei Hagh Yashar, Wu Huapeng, Handroos Heikki

This is a Post-print version of a publication

published by Elsevier

in Fusion Engineering and Design

DOI: $10.1016 /$ j.fusengdes.2020.111532

Copyright of the original publication: ( 2020 Elsevier

Please cite the publication as follows:

Moradkhani, S., Shabbouei Hagh, Y., Wu, H., Handroos, H. (2020). Dynamic analysis and control of a Fusion Reactor Vacuum Vessel Assembly Robot. Fusion Engineering and Design, vol. 154. DOI: 10.1016/j.fusengdes.2020.111532

This is a parallel published version of an original publication. This version can differ from the original published article. 


\section{Journal Pre-proof}

Dynamic analysis and control of a Fusion Reactor Vacuum Vessel Assembly Robot

Shayan Moradkhani (Methodology) (Software) (Formal analysis) (Data curation) (Writing - original draft), Yashar Shabbouei Hagh

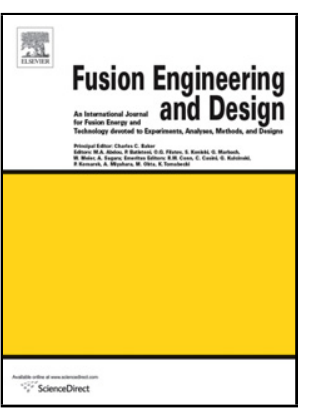
(Conceptualization) (Validation) (Writing - review and editing) (Investigation) (Visualization), Huapeng Wu (Supervision) (Funding acquisition), Heikki Handroos (Project administration)

PII: S0920-3796(20)30080-6

DOI: $\quad$ https://doi.org/10.1016/j.fusengdes.2020.111532

Reference: $\quad$ FUSION 111532

To appear in: $\quad$ Fusion Engineering and Design

Received Date: $\quad 29$ August 2019

Revised Date: $\quad 30$ January 2020

Accepted Date: $\quad 3$ February 2020

Please cite this article as: Moradkhani S, Hagh YS, Wu H, Handroos $\mathrm{H}$, Dynamic analysis and control of a Fusion Reactor Vacuum Vessel Assembly Robot, Fusion Engineering and Design (2020), doi: https://doi.org/10.1016/j.fusengdes.2020.111532

This is a PDF file of an article that has undergone enhancements after acceptance, such as the addition of a cover page and metadata, and formatting for readability, but it is not yet the definitive version of record. This version will undergo additional copyediting, typesetting and review before it is published in its final form, but we are providing this version to give early visibility of the article. Please note that, during the production process, errors may be discovered which could affect the content, and all legal disclaimers that apply to the journal pertain.

(C) 2020 Published by Elsevier. 


\title{
Dynamic analysis and control of a Fusion Reactor Vacuum Vessel Assembly Robot
}

\author{
Shayan Moradkhani ${ }^{\mathrm{a}}$, Yashar Shabbouei Hagh ${ }^{\mathrm{a}}$, Huapeng $\mathrm{Wu}^{\mathrm{a}}$, Heikki Handroos ${ }^{\mathrm{a}}$ \\ ${ }^{a}$ Laboratory of Intelligent Machines, Lappeenranta University of Technology, Lappeenranta, 53850, Finland
}

\begin{abstract}
Highlights
- Developing a parallel robot manipulator for a remote handling application

- Assembly of adjacent vacuum vessel sectors using splice plates

- Designing a robust controller based on non-singular terminal sliding mode control

- The stability of the proposed controller is analyzed by Lyapunov stability criteria
\end{abstract}

The assembly of vacuum vessel (VV) is a vital task for the fusion reactor. This paper introduces simulation of a parallel robot built up for the assembly of fusion reactor CFETR (Chinese Fusion Engineering Test Reactor) to carry out welding of adjacent VV sectors using splice plates, and machining in case some sectors need to be replaced for repair. The kinematics and dynamics equations of the robot are obtained , and a non-singular terminal sliding mode controller is designed which overcomes the drawbacks of the conventional linear sliding mode control. The stability of the system is verified using the Lyapunov stability criteria. The proposed method is tested by defining a trajectory for the robot's endeffector and simulating the multi-body model of the robot.

Keywords: Parallel robot; inverse kinematics analysis; dynamic analysis; Non-singular terminal sliding mode control;

\section{Introduction}

The concept of remote handling makes it possible for operators to carry out the required processes for the assembly of VV using a designed parallel robot without having to be physically present in the reactor, and thus, minimizing the risks involved in exposure to the environment of the fusion reactor. CFETR is the Chinese Fusion Engineering Testing Reactor and is a super conducting magnet Tokamak. In this reactor, the assembly of 1/32 VV is a combination of 4 PSs pre-assembly, processing of PS grooves, assembly of PS, Narrow-gap TIG welding to the PSs, performing non-destructive testing (NDT) to weld joints, and finally some overall measurements. Welding includes connecting the inner and outer shells using an automatic narrow-gap TIG welding. To perform this welding process, a parallel robot was proposed [1]. In addition to the VV assembly, some sectors might have to be replaced for repair. And as serial robots do not inherently show much rigidity in case of adding heavy machining equipment, a parallel manipulator has been considered, which potentially has a better dynamic performance at high speeds and high dynamic loadings [2].

The first proposed model for the VV of ITER (Internationa Thermonuclear Experimental Reactor) was a Penta-WH parallel robot [3]. In this design, there were five linear drives introducing five degrees of freedom, translation of the endeffector and its rotation. The position and orientation of the machining/welding tool mounted on the flange were determined using length measurement of five linear actuators. To increase the dynamic performance of the robot, hydraulic cylinders were used to provide required forces. The inverse kinematics of the robot were carried out, and the workspace of the Penta-WH was obtained using the forward kinematics problem. The dynamic analysis of the system was carried out using ADAMS (a dynamic analysis software). The sixth degree of freedom was accomplished by introducing a carriage moving the robot along a guiding rail.

In a later study, a new design of Penta-WH parallel was proposed for the assembly and repair of the VV of ITER [4]. In this design, the whole robot provided six degrees of freedom. The end-effector had five degrees of freedom, three for the position and two for the rotation respectively. The sixth degree of freedom is for the movement of the robot along the track rail inside of the VV using a servo motor. The actuators implemented in this robot were water hydraulic cylinders because of their higher force density. The inverse kinematics and forward kinematics problem for this robot were solved to determine the workspace of the manipulator. Finally, the designed robot and derived mathematical formulations were tested using lab experiments.

Numerous researches have been done on solving the kinematics and dynamics problems of Stewart-like parallel manipulators. The inverse dynamics problem of the Stewart-platform has been solved using Lagrange and Newton-Euler 
formulations considering the inertias of the cylinders. Linear motors were also used in the control and dynamics analysis of the Stewart-platform. Finally, PID control was used to ensure that the robot tracks a desired trajectory [5]. In another study, a comparison has been made between the results obtained from derived inverse dynamics formulation using Lagrangian formulation and simulation results obtained from dynamic simulation of the Stewart-platform carried out in the ADAMS software [6]. Harib and Sirnivasan [7] have derived the forward kinematics of the Stewart platform using a numerical method. The equations regarding the dynamic analysis of the Stewart platform are also obtained from NewtonEuler formulation for rigid bodies. Ghobakhloo and Azadi [8] obtained the full dynamics of the Stewart platform using Lagrange formulation. In this study, an inverse dynamics control scheme was proposed pushing the robot to track a desired trajectory, and the stability of the control mechanism was verified using Lyapunov criteria.

Different implementations of the PID controller have been applied to control robot manipulators. For instance, [9, 10] introduced a combined version of a PID and (Linear-quadratic regulator) LQR controller, and an optimal fractional order PID for a robotic manipulator, respectively. Using the PID controller in such systems has some disadvantages. Using a PID controller as a linear controller may not guarantee an accurate behavior of nonlinear systems with variable performances. Another main drawback of PID controller is the low robustness of this controller when the operating system faces external disturbances or noises. Sliding mode control (SMC) is an alternative, which has the advantages of the PID such as simple implementation, and on the other hand, covers the drawbacks of the PID. The nonlinear versions of SMC such as terminal SMC (TSMC) [11], non-singular terminal SMC (NTSMC) [12-14], and integral NTSMC [15] are presented and studied for serial link robot manipulators, which have shown unique features compared to linear controllers such as PID.

In this paper, a non-singular terminal sliding mode control is proposed for a parallel manipulator. This controller has the advantages of the conventional SMC such as, simple implementation, robust to external disturbances and fast response. The proposed controller improves the system performance, guarantees finite-time convergence of the states, and evades the singularity problem of the TSMC that causes an unbounded control magnitude. The dynamic model of the robot is obtained using Newton-Euler and Lagrange formulation, and the inverse kinematics model is also presented to provide a real-time simulation.

The rest of the paper is arranged in five sections. Section 2 explains the dynamic of the proposed parallel manipulator, including its inverse kinematics. Section 3 discusses the proposed NTSMC controller. The simulation and results are presented in Section 4 and finally, the paper concludes in Section 5.

\section{Parallel robot model}

In this section, the inverse kinematics and the dynamic equations of the designed system are derived. The final design of the parallel manipulator used in this study is depicted in Figure 1. There are totally seven degrees of freedom for the robot; three translations and rotations of the end-effector and one translation for the carriage that moves along the track rail inside of the VV of CFETR. Lagrange formulation and Newton-Euler methods are used to derive the dynamic equations and to solve the inverse dynamics problem of the robot. The system implemented in this study is a Stewart platform-like manipulator that has a base platform moving along a rail inside of the VV, so unlike the usual Stewart platform design, the base is not fixed to the ground, giving the robot an extra degree of freedom. In this study, however, the reference platform is considered stationary.

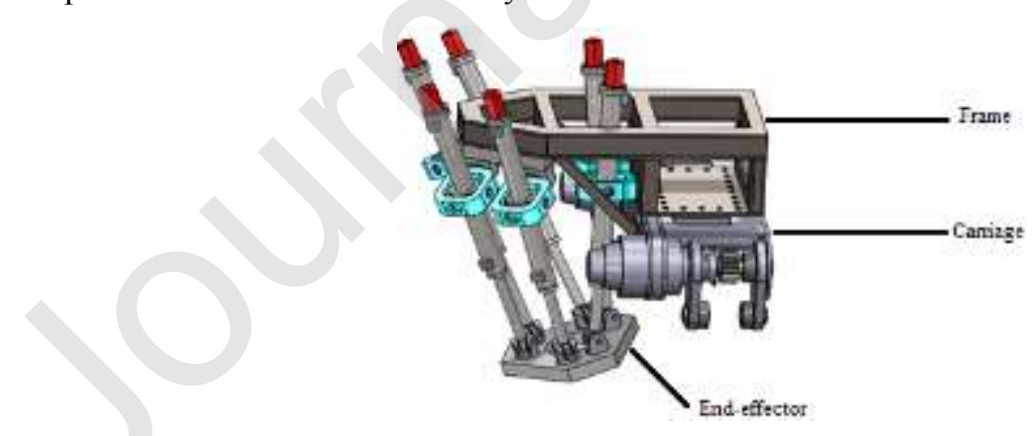

Figure 1.3D model of the parallel manipulator for the assembly of the CFETR reactor.

The robot employed in the assembly of the splice plates inside of the VV moves along a track rail, adding the seventh degree of freedom to the manipulator. The schematic of the robot configuration inside the VV is given in Figure 2. 


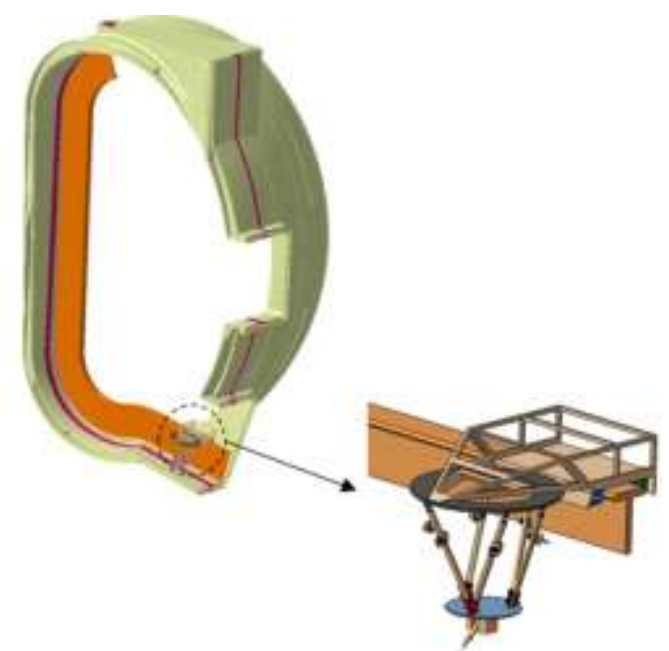

Figure 2. Moving mechanism of the designed parallel robot inside the VV.

\subsection{Inverse Kinematics}

The inverse kinematics problem calculates the required joint configurations for each of the six actuators on the robot in order for the end-effector to track a desired trajectory. In order to determine the orientation and location of the platform in space, six coordinates are needed, and these coordinates are used with respect to the base platform. The first step is to attach one coordinate system $\left(X_{P}-Y_{P}-Z_{P}\right)$ to the center of the platform and another coordinate system $\left(X_{B}-Y_{B}-Z_{B}\right)$ to the center of the base. Vector $P$ denotes the position and the orientation of the platform.

$$
P=\left[\begin{array}{lllll}
x & y & z & \alpha & \beta
\end{array}\right]^{T}
$$

The joint locations on the platform are with respect to the platform coordinate system and the joint locations on the base platform are with respect to the base coordinate system. Figure 3, shows only one leg closed-loop schematic. Euler angles are implemented to define the rotation of the platform with respect to the base, and the rotation matrix is obtained by applying the rotation using this method as follows.

$$
\begin{aligned}
& R_{P}^{B}=R_{z}(\gamma) * R_{y}(\beta) * R_{x}(\alpha) \\
& R_{P}^{B}=\left[\begin{array}{ccc}
c \beta c \gamma & s \alpha s \beta c \gamma-c \alpha s \gamma & c \alpha s \beta c \gamma+s \alpha s \gamma \\
c \beta s \gamma & s \alpha s \beta s \gamma+c \alpha c \gamma & c \alpha s \beta s \gamma-s \alpha c \gamma \\
-s \beta & s \alpha c \beta & c \alpha c \beta
\end{array}\right]
\end{aligned}
$$

In which, $s$ and $c$ refer to the $\sin$ and the $\cos$ functions.

The leg vector of each actuator is obtained as following:

$$
L_{i}=P+R_{P}^{B} * b_{i}-a_{i}, i=0,1, \ldots, 6
$$

and the length of each actuator is obtained as following:

$$
\mathrm{l}_{\mathrm{i}}=\sqrt{L_{x i}^{2}+L_{y i}^{2}+L_{z i}^{2}}
$$




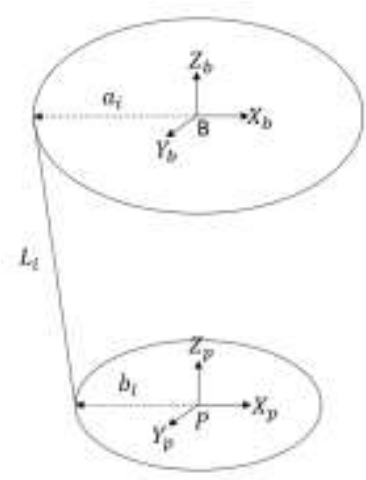

Figure 3. Closed-loop schematic of one leg of the parallel robot.

\subsection{Dynamic analysis of the robot}

In this section, the dynamic analysis of the robot has been carried out using Lagrange formulation and Newton-Euler to derive the dynamic equations related to the links and the platform. The detailed process of deriving the dynamic equations of a Stewart platform is given in [5].

Jacobian matrix could be used to relate the leg velocities to the velocity of the end-effector, and this relation is shown in equation 6 as following:

$$
\dot{l}=J^{-1} * \dot{P}
$$

In the above equation, $\dot{l}$ is a matrix of the leg velocities, and $\dot{P}$ is the velocity of the end-effector. When the determinant of the Jacobian matrix goes to zero, the manipulator goes to singularity condition, and it will not be possible to obtain proper Cartesian forces from the dynamic equations. 7:

The general equation related to the dynamics of the Stewart platform-like robot in this study is presented in equation

$$
M(P) \ddot{P}+B(P, \dot{P}) \dot{P}+K(P)=\left(J^{-1}(P)\right)^{T} F
$$

in which, $\mathrm{M}(\mathrm{P})$ is the inertia matrix, $B(P, \dot{P})$ is Coriolis-Centrifugal, $\mathrm{K}(\mathrm{P})$ is Gravity matrix, $\mathrm{J}(\mathrm{P})$ is Jacobian matrix, and $\mathrm{F}$ is the vector of actuator forces. The inverse dynamics solution takes a desired trajectory of the end-effector and calculates required forces for each actuator to keep the end-effector on the track. Whereas, the forward kinematics solution determines the position and orientation of the end-effector given the actuator forces as input.

\section{The controller design}

In this section, the methodologies of the non-singular terminal sliding mode control (NTSMC) is introduced. Consider the dynamic equation of the robot given in equation 7. The state space representation of this dynamic can be written as:

$$
\begin{aligned}
& \dot{x}_{1}=x_{2} \\
& \dot{x}_{2}=f(x)+b(x) u+d(t)
\end{aligned}
$$

where $\quad x=\left[x_{1}, x_{2}\right]^{T}=[P, \dot{P}]^{T} \quad$ is the state vector, $\mathrm{f}(\mathrm{x})=-M^{-1}(P)(B(P, \dot{P}) \dot{P}+K(P))$ and $\mathrm{b}(\mathrm{x})=M^{-1}(P)\left(J^{-1}(P)\right)^{T}$ are nonlinear functions and the external disturbance is indicated by $\mathrm{d}(\mathrm{t})$ which satisfies ||$d(t) \| \leq l_{d}$ where $l_{d}>0$. The nonlinear NTSM surface is described as:

$$
s=x_{1}+\frac{1}{\beta} x_{2}{ }^{p / q}
$$

in which the $\beta>0$ is the tuning parameter, $p$ and $q$ are the odd integers that should satisfy $1<p / q<2$. Considering the state space representation of equation 8 and the sliding surface of equation 9 , the control signal can be obtained as: 


$$
\begin{aligned}
u= & -b(x)^{-1}\{f(x)+\cdots \\
& \left.+\beta\left(\frac{p}{q}\right) x_{2}{ }^{2-\frac{p}{q}}+\left(l_{d}+\mu\right) \operatorname{sgn}(s)\right\}
\end{aligned}
$$

in which $\mu>0$, and $u$ represents the required control signals for each actuator on the robot.

In order to verify the stability of the proposed controller, consider the Lyapunov function $V=\frac{1}{2} s^{2}$, which should satisfy $\dot{V}=\frac{1}{2}\left(\frac{d}{d t}\right) s^{2} \leq-\mu|s|$, therefore:

$$
\begin{aligned}
\dot{s} s & =\left(\frac{1}{\beta}\right)\left(\frac{p}{q}\right) x_{2}{ }^{\frac{p}{q}-1}\left(d(t) s-\left(L_{d}+\mu\right) \operatorname{sgn}(s) s\right) \\
& \leq-\left(\frac{1}{\beta}\right) \mu\left(\frac{p}{q}\right) x_{2}{ }^{\frac{p}{q}-1}|s|
\end{aligned}
$$

Defining the $\sigma\left(x_{2}\right)=\frac{1}{\beta} \frac{p}{q} \mu x_{2}^{\frac{p}{q}-1}$, it can be deduced that $\dot{s} s \leq \sigma\left(x_{2}\right)|s|$, and since $p$ and $q$ are odd positive constants which $1<p / q<2$, hence, for $x_{2} \neq 0, \sigma\left(x_{2}\right)>0$ and the Lyapunov stability is satisfied. For the case $x_{2}=0$, substituting the control law into the state space representation of the system yields

$$
\begin{aligned}
\dot{x_{2}}= & -\beta\left(\frac{q}{p}\right) x_{2}{ }^{2-\frac{p}{q}}+d(t)+\cdots \\
& -\left(l_{d}+\mu\right) \operatorname{sgn}(s)
\end{aligned}
$$

in which for $x_{2}=0, \dot{x_{2}}=d(t)-\left(l_{d}+\mu\right) \operatorname{sgn}(s)$. It can be seen that for $\mathrm{s}>0$ and $\mathrm{s}<0$ it can be obtained that $\dot{x_{2}} \leq-\mu$ and $\dot{x_{2}} \geq \mu$ respectively. Therefore, $x_{2}=0$ is not an attractor [11]. This completes the proof. It should be noted that the $s g n$ function could cause a chattering phenomenon, which can be evaded by replacing it with sat function.

\section{Simulation and results}

Once the mathematical model of the system has been derived, a trajectory is considered for the robot and is specific to this design only and are considered so that the manipulator does not go into singularity. The simulation for this study is carried out in MATLAB/SIMULINK in order to test the performance of the system mathematically. The simulation starts with the calculation of actuators' lengths through inverse kinematics with given a trajectory. Then, individual lengths are used in the dynamic equation of the system, which is used in the controller, designed in the previous section, to perform the simulation by moving the end-effector. The coordinates of the joint on the moving platform are given in millimeters as:

$$
\begin{aligned}
& b_{1}=\left[\begin{array}{c}
17.37 \\
-98.88 \\
0
\end{array}\right] b_{2}=\left[\begin{array}{c}
76.61 \\
-64.28 \\
0
\end{array}\right] b_{3}=\left[\begin{array}{c}
76.61 \\
64.28 \\
0
\end{array}\right] \\
& b_{4}=\left[\begin{array}{c}
17.37 \\
98.48 \\
0
\end{array}\right] b_{5}=\left[\begin{array}{c}
-93.97 \\
34.2 \\
0
\end{array}\right] b_{6}=\left[\begin{array}{c}
-93.97 \\
-34.2 \\
0
\end{array}\right]
\end{aligned}
$$

The coordinates of the joints on the reference platform are given in millimeters as:

$$
\begin{aligned}
& a_{1}=\left[\begin{array}{c}
-33.45 \\
-227.88 \\
0
\end{array}\right] a_{2}=\left[\begin{array}{c}
214.12 \\
-85 \\
0
\end{array}\right] a_{3}=\left[\begin{array}{c}
214.12 \\
85 \\
0
\end{array}\right] \\
& a_{4}=\left[\begin{array}{c}
-33.45 \\
227.88 \\
0
\end{array}\right] a_{5}=\left[\begin{array}{c}
-180.67 \\
142.88 \\
0
\end{array}\right] a_{6}=\left[\begin{array}{c}
-160.67 \\
-142.88 \\
0
\end{array}\right]
\end{aligned}
$$

The mass of end-effector, rotating part of the leg, and moving part of the leg are approximately $m_{p}=6 \mathrm{~kg}, m_{r}=$ $7 \mathrm{~kg}, m_{m}=3 \mathrm{~kg}$. The trajectory is chosen as:

$$
\begin{aligned}
q & =\left[\begin{array}{llllll}
x & y & z & \alpha & \beta & \gamma
\end{array}\right]^{\prime} \\
& =\left[\begin{array}{llllll}
0 & 0 & 0.75 & 0.2 \sin \left(\frac{\pi t}{2}\right) & 0 & 0
\end{array}\right]^{\prime}
\end{aligned}
$$

In this trajectory, a constant translation is considered and a sinusoidal rotation around the $\mathrm{x}$-axis. The tuning parameters of the NTSM control are considered as $\beta=10, p=7, q=5, l_{d}+\mu=50$. The external disturbance is assumed to be $d(t)=0.1 \sin (3 t)$, and this disturbance 
is applied in the second half of the simulation. The required control signals to the actuators in order for the end-effector to track the trajectory is depicted in Figure 4. Control signals for each actuator to track the desired trajectory.

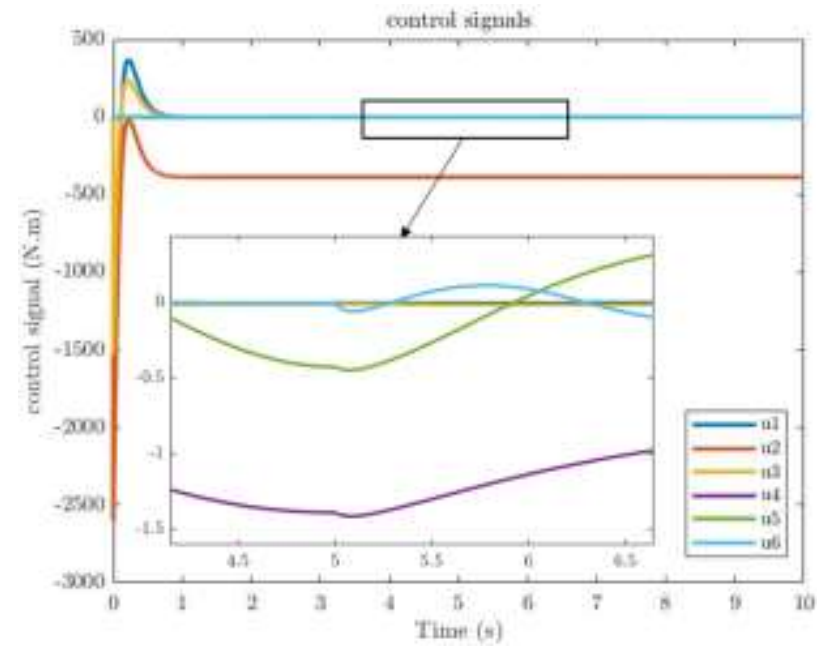

Figure 4. Control signals for each actuator to track the desired trajectory.

Figures 5 to 10 show the actual and desired positions and orientations of the end-effector implementing the NTSMC.

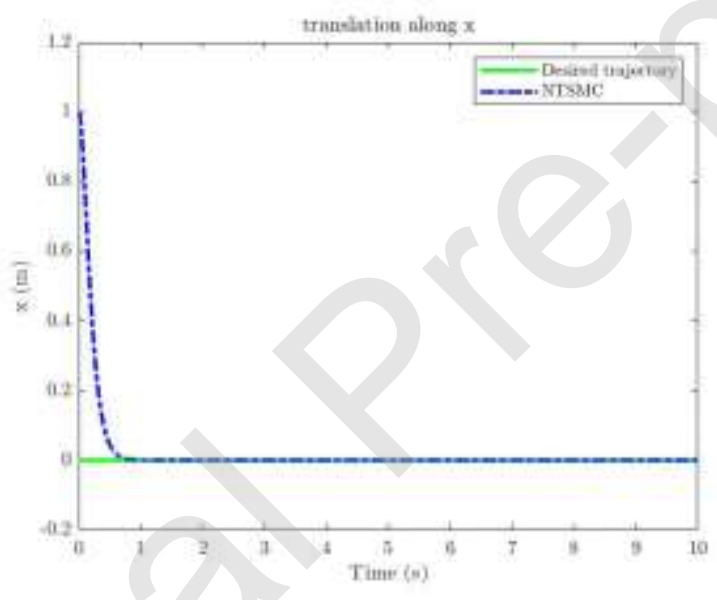

Figure 5. System response with NTSMC for the desired trajectory along x-axis.

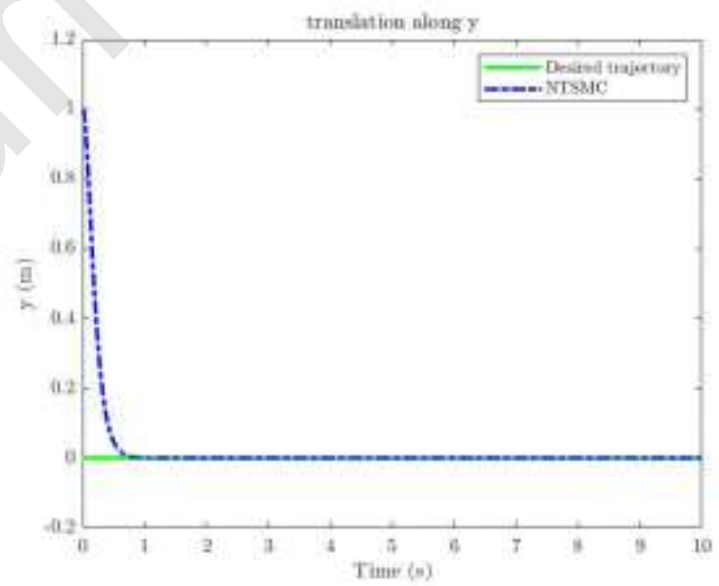

Figure 6. System response with NTSMC for the desired trajectory along y-axis. 


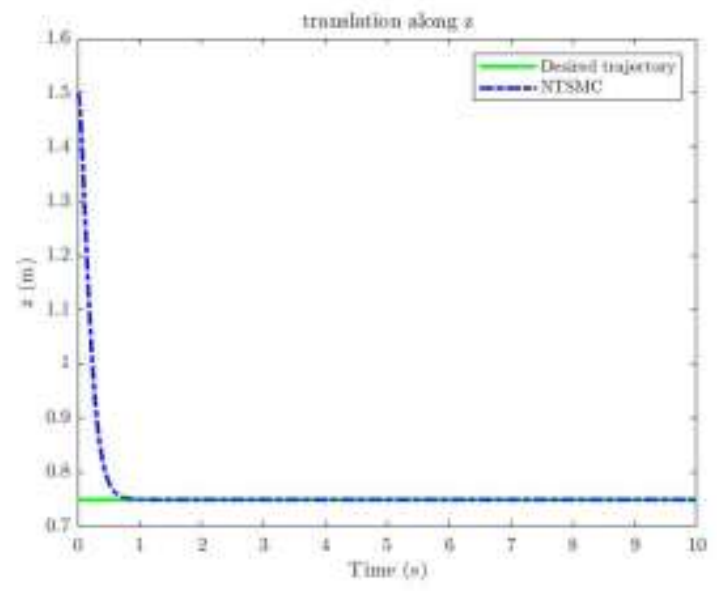

Figure 7. System response with NTSMC for the desired trajectory along z-axis.

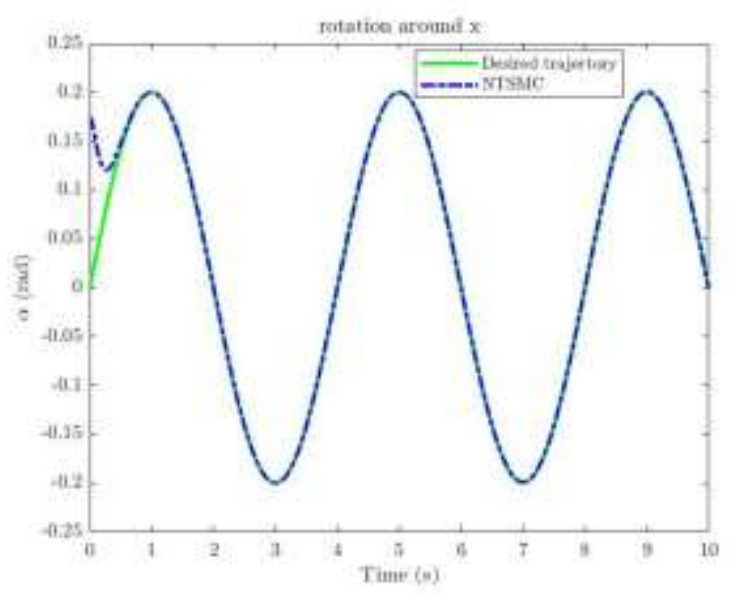

Figure 8. System response with NTSMC for the desired rotation around $\mathrm{x}$-axis.

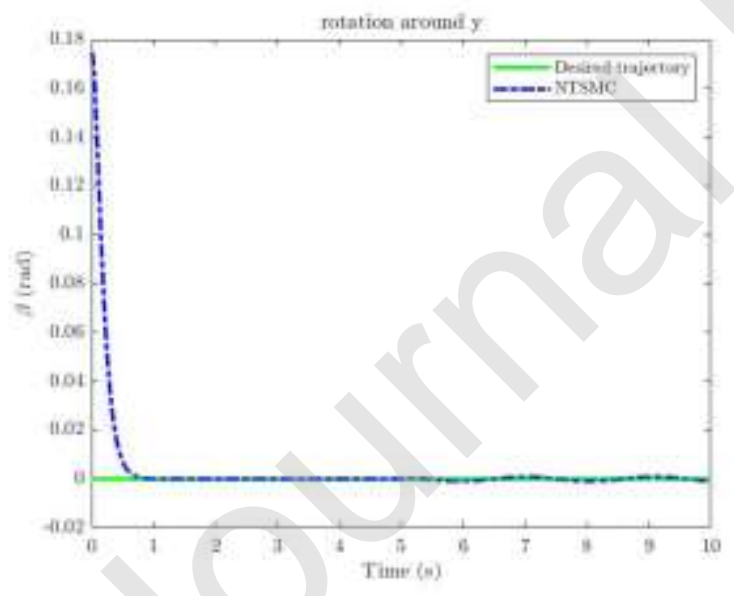

Figure 9. System response with NTSMC for the desired rotation around y-axis. 


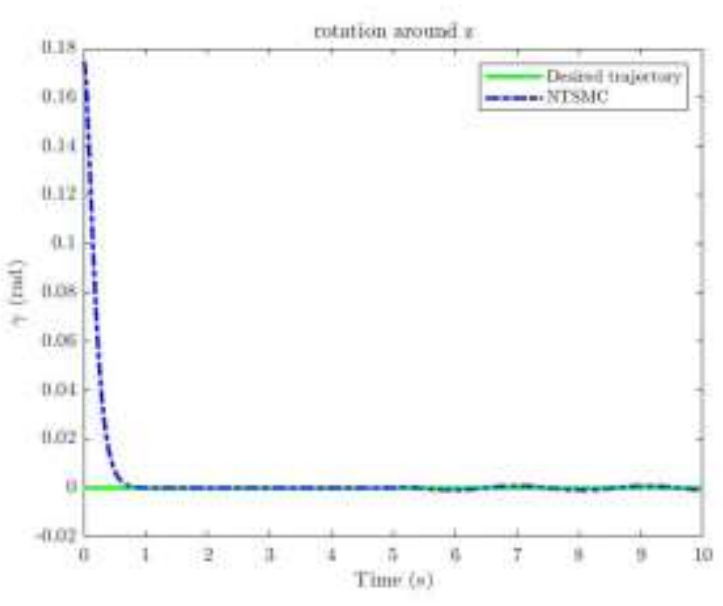

Figure 10. System response with NTSMC for the desired rotation around z-axis.

By observing the translation along $\mathrm{x}$ in Figure 5, it is evident that the controller easily converges the error to zero in around one second, starting from a defined initial value, and the system response matches the desired trajectory afterwards. One unit translation along y-axis was given to the manipulator initially, and it is evident from Error! Reference source not found. that the NTSMC manages to converge the state to zero in almost one second. Along y-axis (Error! Reference source not found.), the desired y displacement reached zero. The initial vertical distance between the end-effector and the reference platform is set to be $1.5 \mathrm{~m}$, but the system was signaled to go to and stay at $0.7 \mathrm{~m}$ over the course of 10-second simulation (Error! Reference source not found.). A sinusoidal signal is considered for the rotation around $\mathrm{x}$-axis ( $\alpha$ angle) in Figure 8. An initial orientation is given to the end-effector around the $\mathrm{x}$-axis in radians. The system starts to track the trajectory with good precision at around 0.5 second into the simulation and stays on track throughout the simulation in the presence of the external disturbance. A slight rotation was applied to the end-effector around the $y$-axis ( $\beta$ angle), and a small orientation was given to the system as well (Figure 6). It can be seen from Figure 9 , that the proposed controller is capable to track the desired trajectory while an external disturbance affect the system from $\mathrm{t}=5 \mathrm{sec}$. As for Figure 10, the rotation around $\mathrm{z}$-axis $(\gamma$ angle), a constant value of zero radians is considered, and the error between the system response and the desired trajectory converges to zero in less than two seconds. The effect of the disturbance from $\mathrm{t}=5 \mathrm{sec}$ and the robustness of the controller is evident in this figure. The results presented so far could be an indication to a predictable remote handling operation. As there is direct supervision of the robot operation, simulation results could ensure that trajectories are being followed and remote handling tasks are being performed with precision.

\section{Conclusion}

In this study, an analysis of the kinematics and dynamics of the designed parallel robot for the assembly of the VV in the CFETR reactor is carried out. Inverse kinematics equations of the manipulator are obtained using the closed-loop geometry of the robot, and full dynamic equations are also obtained to calculate the control signals in order to follow desired a trajectory, which are planned for the manipulator in such a way that it will not go into singularity. A non-singular terminal sliding mode controller is used to control the end-effector's position and orientation. The stability of the proposed controller is proved using the Lyapunov stability criteria. The results of the simulation show that NTSMC is able to stabilize the system throughout the simulation. The aim of implementing the dynamic equations of the robot is to compare the residue between the controlling signal to the actuators in simulation and the actual signals produced by the actuators in the laboratory, in the future, in order to minimize the risks of the sensor malfunctions and faulty signals installed on the robot. The condition monitoring system will be working in parallel with the real system in real-time. To complete the condition monitoring, the forward dynamic simulation of the system will be carried out using commercial software to ensure the reliability of the mathematical equations derived from this study.

Shayan Moradkhani: Methodology, software, formal analysis, Data curation, writing original draft

Yashar Shabbouei Hagh: Conceptualization, validation, writing-review and editing, investigation, visualization

Huapeng Wu: Supervision, funding acquisition

Heikki Handroos: Project administration 


\section{Declaration of interests}

The authors declare that they have no known competing financial interests or personal relationships that could have appeared to influence the work reported in this paper.

\section{Acknowledgments}

The authors would like to appreciate the support of Eurofusion Education for this study and whomever else that contributed to this work.

\section{References}

[1] Liu Z, Wu J, Fan X, Ma J, Wang R. A study on assembly technology of the CFETR 1/32 Vacuum Vessel. Fusion Engineering and Design 2018; 128: 101-6.

[2] Wu H, Handroos H, Kovanen J, et al. Design of parallel intersector weld/cut robot for machining processes in ITER vacuum vessel. Fusion Engineering and Design 2003; 69(1-4): 327-31.

[3] Wu H, Handroos H, Kovanen J, et al. Design of parallel intersector weld/cut robot for machining processes in ITER vacuum vessel. Fusion Engineering and Design 2003; 69(1-4): 327-31

[https://doi.org/10.1016/S0920-3796(03)00066-8]

[4] Wu H, Handroos H, Pessi P, Kilkki J, Jones L. Development and control towards a parallel water hydraulic weld/cut robot for machining processes in ITER vacuum vessel. Fusion Engineering and Design 2005; 75-79: 625-31

[https://doi.org/10.1016/j.fusengdes.2005.06.304]

[5] Şumnu A, Güzelbey İH, Çakir MV. Simulation and PID control of a Stewart platform with linear motor. J Mech Sci Technol 2017; 31(1): 345-56

[https://doi.org/10.1007/s12206-016-1238-7]

[6] Hajimirzaalian H, Moosavi H, Massah M. Dynamics analysis and simulation of parallel robot Stewart platform. In: Dynamics analysis and simulation of parallel robot Stewart platform; 2010; 472-7.

[7] Harib K, Srinivasan K. Kinematic and dynamic analysis of Stewart platform-based machine tool structures. Robotica 2003; 21(5): 541-54

[https://doi.org/10.1017/S0263574703005046]

[8] Ghobakhloo A, Eghtesad M, Azadi M. Position control of a stewart-gough platform using inverse dynamics method with full dynamics. In: Position control of a stewart-gough platform using inverse dynamics method with full dynamics; 2006. IEEE; 50-5.

[9] Asl RM, Mahdoudi A, Pourabdollah E, Klančar G. Combined PID and LQR controller using optimized fuzzy rules. Soft Computing 2019; 23(13): 5143-55.

[10] Asl RM, Pourabdollah E, Salmani M. Optimal fractional order PID for a robotic manipulator using colliding bodies design. Soft Computing 2018; 22(14): 4647-59.

[11] Feng Y, Yu X, Han F. On nonsingular terminal sliding-mode control of nonlinear systems. Automatica 2013; 49(6): 1715-22.

[12] Hagh YS, Asl RM, Cocquempot V. A hybrid robust fault tolerant control based on adaptive joint unscented kalman filter. ISA transactions 2017; 66: 262-74.

[13] Asl RM, Hagh YS, Palm R. Robust control by adaptive non-singular terminal sliding mode. Engineering Applications of Artificial Intelligence 2017; 59: 205-17.

[14] Asl RM, Hagh YS, Handroos H. Adaptive extended kalman filter designing based on non-singular fast terminal sliding mode control for robotic manipulators. In: Adaptive extended kalman filter designing based on non-singular fast terminal sliding mode control for robotic manipulators; 2017; 1670-5.

[15] Asl RM, Hagh YS, Palm R, Handroos H. Integral non-singular terminal sliding mode controller for nth-order nonlinear systems. IEEE Access 2019. 